\section{Response to: 'Novel NLRP12 variant presenting with familial cold autoimmunity syndrome phenotype' by Gupta et al}

We read with great interest the letter by Aggarwal. ${ }^{1}$ Her case is one of early-onset autoinflammatory disease.

Her case is not directly similar to any of the 187 cases we described in our paper on undefined autoinflammatory diseases. ${ }^{2}$

Given the finding of novel potentially pathogenic NLRP12variants in homozygosity, we think the authors have most likely identified a hitherto unknown cause of this child's symptoms. As such, we recommend the case with all its details to be submitted for publication in a scientific journal.

\section{Charlotte Eijkelboom $\odot,{ }^{1}$ Nienke M Ter Haar, ${ }^{2}$ Joost Frenkel, ${ }^{3}$ Marco Gattorno ${ }^{4}{ }^{4}$ \\ ${ }^{1}$ Paediatrics, Universitair Medisch Centrum Utrecht, Utrecht, The Netherlands ${ }^{2}$ Laboratory for Translational Immunology, UMC, Utrecht, The Netherlands ${ }^{3}$ General Pediatrics, University Medical Center Utrecht, Utrecht, The Netherlands ${ }^{4}$ Paediatric Rheumatology, Istituto Giannina Gaslini, Genova, Italy}

Correspondence to Dr Joost Frenkel, General Pediatrics, University Medical Center Utrecht, Utrecht 3584, The Netherlands; j.frenkel@umcutrecht.nl

Handling editor Josef S Smolen

Funding The authors have not declared a specific grant for this research from any funding agency in the public, commercial or not-for-profit sectors.

Competing interests None declared.
Patient consent for publication Not required.

Provenance and peer review Commissioned; internally peer reviewed.

(c) Author(s) (or their employer(s)) 2021. No commercial re-use. See rights and permissions. Published by BMJ.

\section{Check for updates}

To cite Eijkelboom C, Ter Haar NM, Frenkel J, et al. Ann Rheum Dis 2021;80:e118.

Received 27 August 2019

Accepted 27 August 2019

Published Online First 6 September 2019

\section{Linked}

- http://dx.doi.org/10.1136/annrheumdis-2019-216158

Ann Rheum Dis 2021:80:e118. doi:10.1136/annrheumdis-2019-216184

\section{ORCID iDs}

Charlotte Eijkelboom http://orcid.org/0000-0001-6202-3628

Marco Gattorno http://orcid.org/0000-0003-0704-1916

\section{REFERENCES}

1 Gupta L, Ahmed S, Singh B. Novel NLRP12 variant presenting with familial cold autoimmunity syndrome phenotype. Ann Rheum Dis 2021;80:e117.

2 Ter Haar NM, Eijkelboom C, Cantarini L, et al. Clinical characteristics and genetic analyses of 187 patients with undefined autoinflammatory diseases. Ann Rheum Dis 2019;78:1405-11. 